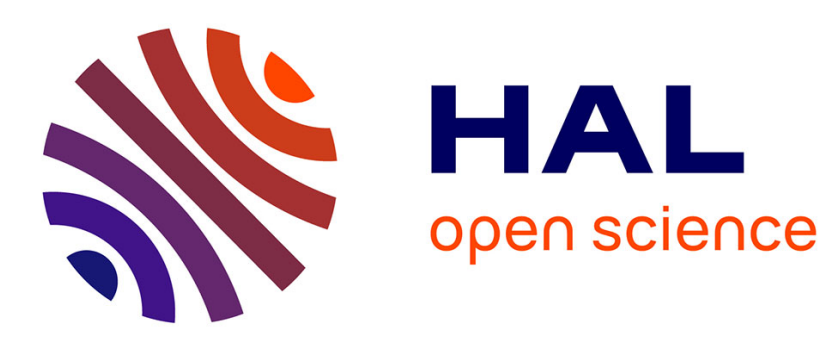

\title{
Coupled modes semiclassical treatment of nonadiabatic transitions
}

\author{
Marc Boiron, Maurice Lombardi, Laurent Wiesenfeld
}

\section{To cite this version:}

Marc Boiron, Maurice Lombardi, Laurent Wiesenfeld. Coupled modes semiclassical treatment of nonadiabatic transitions. Journal of Physics A: Mathematical and Theoretical, 1997, 30, pp.39073926. 10.1088/0305-4470/30/11/019 . hal-00972654

\section{HAL Id: hal-00972654 https://hal.science/hal-00972654}

Submitted on 3 Apr 2014

HAL is a multi-disciplinary open access archive for the deposit and dissemination of scientific research documents, whether they are published or not. The documents may come from teaching and research institutions in France or abroad, or from public or private research centers.
L'archive ouverte pluridisciplinaire HAL, est destinée au dépôt et à la diffusion de documents scientifiques de niveau recherche, publiés ou non, émanant des établissements d'enseignement et de recherche français ou étrangers, des laboratoires publics ou privés. 


\title{
Coupled modes semiclassical treatment of nonadiabatic transitions
}

\author{
Marc Boiron, Maurice Lombardi and Laurent Wiesenfeld \\ Laboratoire de Spectrométrie Physique, Université Joseph-Fourier-Grenoble, BP87, F-38402 \\ Saint-Martin-d'Hères Cédex, France
}

Received 14 October 1996, in final form 12 March 1997

\begin{abstract}
We analyse the Schrödinger wave equation of a two-level or spinorial Hamiltonian, from a classical point of view. An iterative scheme, the coupled mode semiclassical formalism, is proposed, allowing us to deal with the nonadiabatic transfer. As the WKB expansion, it allows the one-dimensional Schrödinger equation to be integrated by successive quadratures.

Finally, we show that time-dependent information can be drawn from the previous, purely stationary, analysis by extending the notion of group velocity. The proposed formalism is thus coherent with an image of multiple trajectories, conforming more to physical behaviour than a single trajectory.
\end{abstract}

\section{Introduction}

The aim of the present paper is to derive a semiclassical formalism for a spinorial Hamiltonian. Our primary interest for such a study is vibrational quantum chaos generated by nonadiabatic couplings between electronic energy levels in molecules. Recent spectroscopic studies [1] of the $\mathrm{NO}_{2}$ molecule, through statistical analysis of the energy spectra, clearly exhibit transition to chaos in the vicinity of the conical intersection of their two first-electronic energy levels, that is, where nonadiabatic couplings are highest. The socalled quantum chaos, based on random matrix theory [2] and statistical analysis, has been related to classical chaos [3], even though contrasting examples have been exhibited [4]. For mixed phase space classical Hamiltonians, research is still in progress. In the case of two-level systems, the situation is worse as none of the previous results can be applied. Our aim is then to obtain a classical limit of Hamiltonians of such systems, modelling molecules in coupled electronic states. Within classical mechanics, chaos, by means of standard tools, can then be studied. The scope of the following theoretical analysis has been restricted: only one-dimensional two-level Hamiltonians in the case of an avoided crossing will be considered. Results of comparative studies of classical and quantum chaos will be given elsewhere.

The classical limit of two-level or more generally, spinorial Hamiltonians has a long history and is still a subject of current research. It begins with the well known LandauZener (LZ) formula [5] for the two-level case. Zener, through a strongly intuitive approach, considered that in the classical regime, the time-dependent wavefunction is a single twocomponent wavepacket with a prescribed motion. In the vicinity of the avoided crossing of the adiabatic potentials, diabatic potentials are linearly approximated and the diabatic coupling is taken as constant. A set of coupled first-order time-dependent differential 
equations for the probability amplitude of each level, is then laid down thanks to physical arguments. Its asymptotic study gives the probability transfer as a function of the speed of the wavepacket and other relevant physical parameters. Subsequent analysis of Stückelberg leads to the same formula but is founded on more rigorous bases. This author considers the stationary adiabatic wavefunctions far from the avoided crossing point and connects them through the complex plane similar to how usual WKB wavefunctions are related through a turning point. This method has been analysed in detail by Thorson et al [6] for general potentials. They pointed out that severe restrictive conditions should be satisfied in order to rigorously derive the LZ formula. Moreover, the full $2 \times 2$ scattering matrix entails an undetermined phase on the off-diagonal elements. Despite that, it works surprisingly well when compared with exact numerical computation. Child [7] considers the problem in the restrictive frame of linear diabatic potentials coupled by a constant, for energies far from the crossing point. He derived a full expression for the scattering matrix.

The appealing approach, initiated by Zener, of the classical limit through a single wavepacket moving on two electronic surfaces has since been generalized to several degrees of freedom and several surfaces. The derived formalism is usually named classical trajectory approximation $[8,9]$. An improved version of it, known as mean trajectory approximation or hemiquantal treatment, has been proposed [10] where feedback between probability amplitudes and motion of the wavepacket is taken into account. A formalism of that type (called 'one centre approximation'), was used by us to study diffusive chaos in two-level systems [11]. For more complete references and other proposed formalisms see [12]. The validity of trajectory approaches was carefully analysed in the two-level one-dimensional case [13]. It was shown, from an analysis of the stationary Schrödinger equation, that such an image can be applied to experimental results provided that the condition of ' \pm separation' is fulfilled, that is, the difference between the momenta corresponding to the two adiabatic levels has to be small compared with their sum. Therefore, such approaches are not satisfying, particularly when one deals with well separated adiabatic potentials out of the avoided crossing points at low energy. In that case, the ' \pm separation' condition is not satisfied, the components of the wavepacket move at different speeds and a classical trajectory approximation cannot be valid. Moreover, the encounter of the avoided crossing point by a wavepacket causes its splitting. In the vibrational case, successive splittings are expected as time evolves.

Even if a single-trajectory formalism cannot give the right description of the behaviour of a wavepacket in several coupled potentials, it does not mean, however, that the usual classical condition, that is, small de Broglie wavelength with respect to the typical length of the potentials, is not satisfied. From this remark, we try to derive a semiclassical stationary wavefunction of a two-level Hamiltonian in the spirit of the WKB expansion [14] in the coupled as well as in the uncoupled region on the potentials. We would qualify such an approach as 'weak' because no assumptions are made on the solution of the problem, in contrast to the 'strong' approach of a single trajectory formalism, where a restrictive frame is imposed to derive the classical limit.

Other semiclassical theories of spinorial systems, not based on single trajectory, have been proposed. The use of Wigner transform [11] and the limit $\hbar$ going to zero, leads to the adiabatic approximation. It is not satisfying, as nonadiabatic couplings are lost. Littlejohn [15] and others [16] use a preliminary diagonalization of the spinorial Hamiltonian operator in the space of position/momentum operators, then transform the eigenvalues in their Weyl symbol and expand in $\hbar$. It leads to the adiabatic approximation but we take into account Berry's phase around a singularity such as a conical intersection. A subsequent work is based on the use of normal forms [17] in the vicinity of a one-dimensional avoided 
crossing. Zhu et al [18] considered the problem in the same frame as Child did. By means of analysis in the complex plane using the Stokes phenomenon, they derived the elements of the scattering matrix as a converging series. Let us also cite the work of Voronin et al [19] addressing semiclassical quantization of Jahn-Teller molecules. Their results are valid under a condition similar to the ' \pm separation'.

Let us explain how this paper is divided. In the following section 2, we first pose our frame of study. The results of the usual semiclassical method, by means of an expansion, are reviewed when applied to Hamiltonian (1), focusing on probability transfer. Section 3 occupies a central place in this paper: a new semiclassical iterative scheme is derived, applying to spinorial one-dimensional Hamiltonians as well as ordinary ones. Comparison between approximate and exact wavefunctions shows that this scheme is successful when nonadiabatic transitions occur. Three applications are then developed in section 4: reflection of a wavepacket over a potential barrier for a scalar Hamiltonian, comparison with the LZ formula and derivation of generalized group velocities. This last application, the most important, allows us to recover time-dependent information from a time-independent study and shows that our analysis, unlike previous ones, is consistent with the splitting of wavepackets at the encounter with the crossing point.

\section{Semiclassical approximation and probability transfer}

\subsection{The model}

The starting point for our semiclassical study of vibrational behaviour of two-level systems is the following nuclear Hamiltonian obtained by projection in the so-called diabatic basis [20]:

$$
\hat{H}_{D}=\left(\begin{array}{cc}
\hat{P}^{2} / 2 m & 0 \\
0 & \hat{P}^{2} / 2 m
\end{array}\right)+\left(\begin{array}{cc}
V_{1}(\hat{X}) & V \\
V & V_{2}(\hat{X})
\end{array}\right) .
$$

We restrict our study to one particle of reduced mass $m$ in the one-dimensional diabatic potentials $V_{1}$ and $V_{2}$. $\hat{X}$ and $\hat{P}$ are the usual position and momentum operators. $V$ is the diabatic coupling potential term, taken as real and constant in order to simplify calculations. Extension to the general case is straightforward as explained in the conclusion.

The usual projection basis is the adiabatic one, made of the electronic states obtained as eigenvectors of the electronic part of the exact molecular Hamiltonian. By means of these states, a nuclear Hamiltonian can be derived. Couplings between amplitudes of different levels is then essentially of a kinetic nature. Thus, this method is the natural way to go to the Born-Oppenheimer approximation that consists of neglecting these couplings. It is physically based on the difference of speed between electrons and nuclei owing to the high ratio of their masses. Our aim being to take into account such couplings, the diabatic basis is then a more convenient starting point as the couplings are described by positiondependent terms, abusively called potential. Such a basis is obtained either by a proper rotation of the adiabatic basis or in the frame of the so-called crude-adiabatic approximation [21]. In any case, it is possible, at least approximately, to recover the expression of the Hamiltonian in the adiabatic basis by means of the rotation diagonalizing the potential part of Hamiltonian (1). The resulting matrix of space-dependent eigenvectors does not commute with the kinetic part of $\hat{H}_{D}$ and gives rise to kinetic couplings as well as potential couplings. From Hamiltonian (1), this procedure leads to the following Hamiltonian:

$$
\hat{H}_{A}=\left(\begin{array}{cc}
\hat{P}^{2} / 2 m & 0 \\
0 & \hat{P}^{2} / 2 m
\end{array}\right)+\left(\begin{array}{cc}
U_{1}(\hat{X}) & 0 \\
0 & U_{2}(\hat{X})
\end{array}\right)+K(\hat{X})\left(\begin{array}{cc}
0 & -\hat{P} \\
\hat{P} & 0
\end{array}\right)
$$


where

$$
\begin{aligned}
& U_{1,2}=\frac{V_{1}+V_{2} \pm \sqrt{\left(V_{1}-V_{2}\right)^{2}+4 V^{2}}}{2} \\
& K=\frac{\mathrm{i} \hbar}{m} \frac{V}{\left(U_{1}-U_{2}\right)^{2}} \frac{\mathrm{d}\left(V_{1}-V_{2}\right)}{\mathrm{d} \hat{X}} .
\end{aligned}
$$

Potential terms arising in this change of basis have been omitted, they are not essential to our discussion. In practice, they are often neglected. $\hat{H}_{A}$ does have the form of the molecular Hamiltonian projected in the adiabatic basis, restricted to two levels.

In the adiabatic or Born-Oppenheimer approximation, only diagonal terms are retained. It allows us to deal with two uncoupled adiabatic Hamiltonians:

$$
\hat{H}_{1,2}=\frac{\hat{P}^{2}}{2 m}+U_{1,2}(\hat{X}) .
$$

$U_{1}$ and $U_{2}$ are recognized as the usual electronic surfaces, that is, the potentials of interaction of the nuclei in each level respectively. From (2) and (4), it is easy to realize that such an approximation is valid for low lying states $(\langle\hat{P}\rangle$ small $)$ and in the case of sufficient separation of the electronic surfaces $\left(U_{1}-U_{2}\right.$ large). Throughout the paper, the adiabatic approximation (5) will be referred to, hence this brief summary.

The situations we will deal with, do not reduce to this approximation, at least not on the whole range of space of interest. The breakdown of such an approximation is encountered in practice at crossing points of the adiabatic potentials $\left(V_{1}=V_{2}\right)$ where $K$ of (2) reaches its maximum value: $\frac{i \hbar}{m} \frac{1}{4 V} \frac{\mathrm{d}\left(V_{1}-V_{2}\right)}{\mathrm{d} \hat{X}}$. Around such a point, for a sufficient energy, the two components of the adiabatic nuclear wavefunction are appreciably coupled; therefore, transfer of probability between them is expected to occur. That quantity, depending on the energy of the incident wave and the parameters of the potentials, is the most important in qualitatively understanding the behaviour of the system. If it is equal to zero, it simply means that the adiabatic approximation is valid. Far from the crossing point, the adiabatic as well as the diabatic potentials separate, decreasing the value of $K$, thus, making the adiabatic approximation valid locally. Such a generic situation of coexistence of adiabatic and coupling regimes is illustrated in figure 1. Diabatic and adiabatic potentials, in reduced units $\hbar=m=1$, are plotted as a function of space for two values of the coupling $V$. At the crossing point of $V_{1}$ and $V_{2}, U_{1}$ and $U_{2}$ avoid crossing, hence the wording 'avoided crossing' also used to name, from the adiabatic point of view, the interval where probability transfer occurs. Note that in the particular case of figure 1, the adiabatic regime is forced for large $\|x\|$-that is roughly outside the interval represented in figure 1 -since the potentials there are flat $\dagger$, which involves, from (4), that $K=0$.

\subsection{The usual WKB expansion}

Let us write the time-independent Schrödinger equation from Hamiltonian (1) in the space representation:

$$
\left(\begin{array}{cc}
-\frac{\hbar^{2}}{2 m} \frac{\mathrm{d}^{2}}{\mathrm{~d} x^{2}}+V_{1}(x) & V \\
V & -\frac{\hbar^{2}}{2 m} \frac{\mathrm{d}^{2}}{\mathrm{~d} x^{2}}+V_{2}(x)
\end{array}\right)\left(\begin{array}{c}
\alpha(x) \\
\beta(x)
\end{array}\right)=E\left(\begin{array}{c}
\alpha(x) \\
\beta(x)
\end{array}\right)
$$

$\dagger$ The potentials were taken, respectively, as the functions $V_{1}=-10 \tanh ((x+16) / 10)-4$ and $V_{2}=-8 \mathrm{e}^{-(x / 20)^{2}}$. 


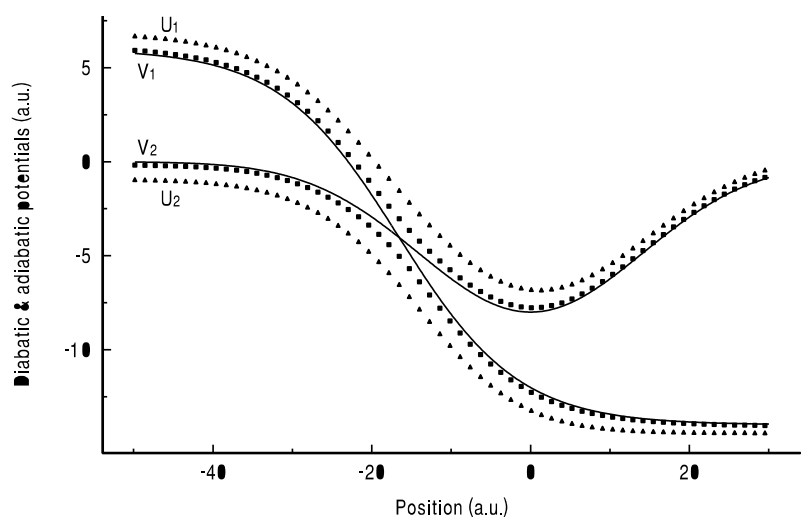

Figure 1. Potentials used for the study of quantal and semiclassical wavefunctions in two-level systems in reduced units: $\hbar=m=1$. The full curves are the diabatic potentials $V_{1}$ and $V_{2}$. The scattered curves are the adiabatic potentials $U_{1}$ and $U_{2}$ in the case of diabatic coupling $V$ equal to $3(\mathbf{\Lambda})$ or $1.5(\mathbf{\square})$.

satisfied by a spinorial wavefunction $\left(\begin{array}{l}\alpha \\ \beta\end{array}\right)$. The most direct way to get a semiclassical approximation would be to substitute the following ansatz for the wavefunctions in (6):

$$
\alpha(x)=A(x) \mathrm{e}^{\mathrm{i} S(x) / \hbar} \quad \text { and } \quad \beta(x)=a(x) \mathrm{e}^{\mathrm{i} \sigma(x) / \hbar}
$$

where $A, a, S, \sigma$ are real quantities. Straightforward replacing of expression (7) in (6) does not work because phase terms, i.e. $\mathrm{e}^{\mathrm{i}(S-\sigma) / \hbar}$ remain in the equations. We will eliminate one component, say $\beta$, from (6). One obtains:

$$
\begin{aligned}
-\frac{\hbar^{4}}{4 m^{2}} \frac{\mathrm{d}^{4}}{\mathrm{~d} x^{4}} \alpha(x) & -\frac{\hbar^{2}}{2 m}\left(2 E-V_{1}(x)-V_{2}(x)\right) \frac{\mathrm{d}^{2}}{\mathrm{~d} x^{2}} \alpha(x)+\frac{\hbar^{2}}{m} V_{1}^{\prime}(x) \frac{\mathrm{d}}{\mathrm{d} x} \alpha(x) \\
+ & {\left[\frac{\hbar^{2}}{2 m} V_{1}^{\prime \prime}(x)+V^{2}-\left(E-V_{1}(x)\right)\left(E-V_{2}(x)\right)\right] \alpha(x)=0 . }
\end{aligned}
$$

The prime (') denotes derivation with respect to space. Substitution of the ansatz form (7) of $\alpha$ is made into (8) as well as separation into real and imaginary parts. In contrast to the usual case of the second-order Schrödinger equation, the fourth-order derivative gives rise to mixed terms of amplitude and phase, and introduces phase terms with derivatives of order higher than one. In order to get an Hamilton-Jacobi-like equation, i.e. a first-order equation for $S$ uncoupled from $A$, it would be necessary to discard these extra terms. This requires many conditions to be fulfilled and, finally, quite uselessly complicates the way to proceed further.

Giving up this approach, we resort to an expansion method to get a semiclassical approximation. The usual ordering parameter is $\hbar[14,22]$, but, as quoted in [23], in the spinorial case, $\hbar$ is not a well suited parameter. We then proceed using the method stated by Littlejohn [17], a systematic way of making a semiclassical expansion in the sense that no $a$ priori parameter is required. It consists of introducing an extra $\epsilon$ ordering parameter in the ansatz of the semiclassical wavefunction as:

$$
\alpha=\mathrm{e}^{\mathrm{i} S / \epsilon} \quad \text { and } \quad S=S_{0}+\epsilon S_{1}+\epsilon^{2} S_{2}+\cdots
$$

as well as in the wave equation, here (8), through the substitution:

$$
\frac{\mathrm{d}}{\mathrm{d} x} \longrightarrow \epsilon \frac{\mathrm{d}}{\mathrm{d} x}
$$


Once we obtain a (truncated) expression of $S, \epsilon$ is restored to unity.

In our case, straightforward calculations show that $S_{0}$ satisfies the following HamiltonJacobi equation:

$$
\begin{gathered}
J_{4}(x, p)=-\frac{p^{4}}{4}+\frac{2 E-V_{1}(x)-V_{2}(x)}{2} p^{2}+\mathrm{i} V_{1}^{\prime}(x) p+\frac{V_{1}^{\prime \prime}(x)}{2} \\
+V^{2}-\left(E-V_{1}(x)\right)\left(E-V_{2}(x)\right)=0
\end{gathered}
$$

with $p=S_{0}^{\prime}$, and where we have used 'atomic' units $(\hbar=m=1)$. The variable $p$ will be called momentum by analogy to the usual case. $J_{4}$ is thus a complex HamiltonJacobi function. The first order $S_{1}$ in the expansion satisfies the following equation, written conveniently as a function of $J_{4}$ :

$$
\frac{1}{2 \mathrm{i}} \frac{\partial^{2} J_{4}}{\partial p^{2}}\left(x, S_{0}^{\prime}\right) S_{0}^{\prime \prime}+\frac{\partial J_{4}}{\partial p}\left(x, S_{0}^{\prime}\right) S_{1}^{\prime}=0 .
$$

We restrict the expansion to that order. Writing $v=\partial J_{4} / \partial p$, the resolution of (10), (11) leads to the expression of the semiclassical wavefunction:

$$
\alpha(x)=\exp \left(\mathrm{i} \int^{x} p \mathrm{~d} \xi-\int^{x}\left(\frac{\partial v}{\partial p}\right) \frac{p^{\prime}}{2 v} \mathrm{~d} \xi\right) .
$$

$p(x)$ is a root of the polynomial (10) which has one purely imaginary coefficient, it is thus complex valued. It can be conveniently calculated by integration of the complex equation:

$$
\frac{\mathrm{d} p}{\mathrm{~d} x}=\frac{-\partial J_{4} / \partial x}{\partial J_{4} / \partial p}
$$

obtained by differentiating (10), which allows us to 'follow' continuously a particular root as a function of the varying parameter $x$.

Figure 2 displays the comparison between the exact wavefunction and its corresponding semiclassical approximation given by (12) by means of potentials of figure 1 . The following observations are made in general: for a given value of the energy $E$ and a sufficiently strong diabatic coupling $V$, the semiclassical wavefunction achieves to fit accurately the exact one on the whole integration domain. It can be observed for example with the following values: $E=15$ and $V=3$ (result not represented). However, as $V$ decreases with fixed $E$ : figure $2(a)$ where $E=15$ and $V=1.5$, or $E$ increases with fixed $V$ : figure $2(b)$ where $E=60$ and $V=3$, discrepancies occur between these two functions. These discrepancies begin in the vicinity of the crossing point and remain as integration progresses into $x>0$. Corresponding studies of the exact wavefunctions in the adiabatic basis have also been done. As may generally be noted, the semiclassical approximation fails when the probability transfer is not weak. As explained in the introduction, this transfer is the main object of our semiclassical study, thus this new semiclassical scheme does not allow us to treat semiclassically nonadiabatic transitions.

Further numerical studies of the accessible phase space given by the Hamilton-Jacobi equation (10), that is, the roots of this equation as a function of space, leads to the following observations: when two momenta, i.e. two roots, approach each other more and more at some position through a decrease in the diabatic coupling $V$ or an increase in the energy $E$, the semiclassical wavefunction exhibits increasing difference with the exact wavefunction. At the same time, $V \rightarrow 0$ or $E \rightarrow \infty$ causes the probability transfer to increase.

This correlation between near degeneracy and failure of the semiclassical approximation can be explained by considering the condition of validity of the expansion in $\epsilon$ :

$$
\left|\epsilon S_{1}^{\prime}\right| \ll\left|S_{0}^{\prime}\right| \text {. }
$$



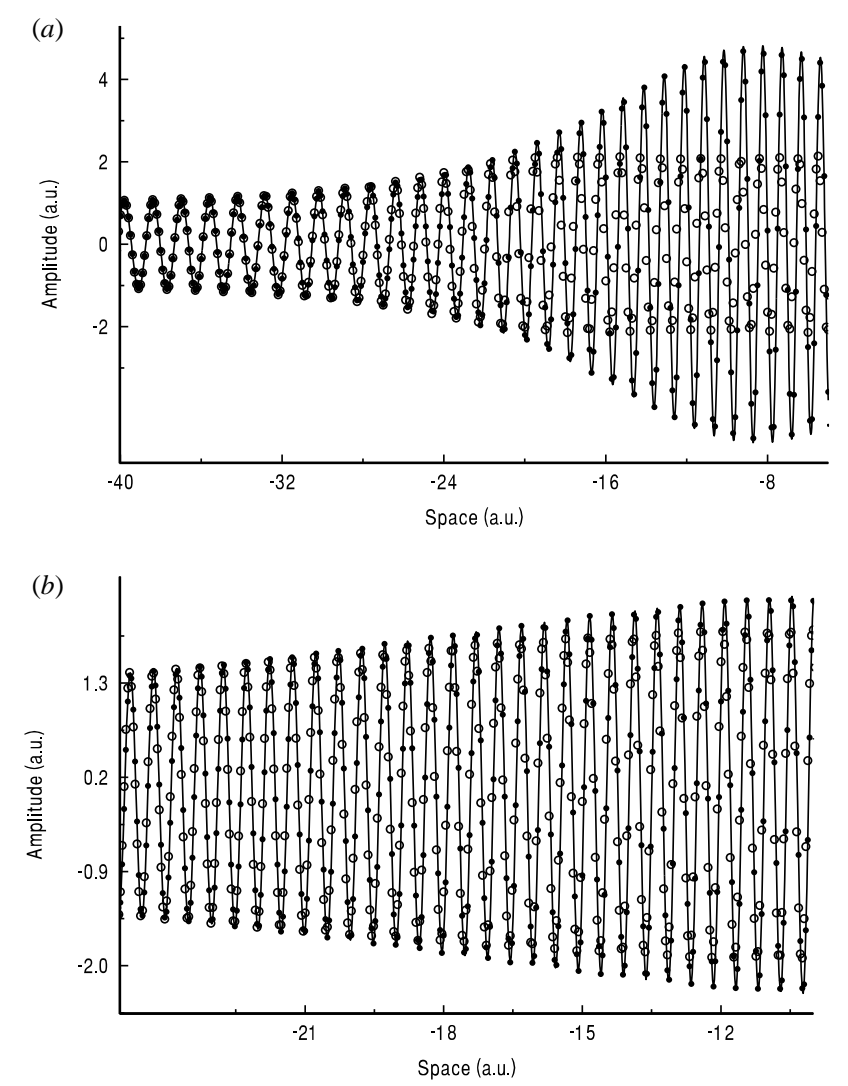

Figure 2. The exact wavefunction (full curve) is compared with the WKB or the semiclassical mode wavefunction $(O)$ and the coupled mode wavefunction $(\bullet)$, in the potential of figure 1 . In the cases illustrated here, the parameters $E$ and $V$ are chosen such that nonadiabatic transfer occurs. (a) $E=15$ and $V=1.5$, (b) $E=60$ and $V=3$. The WKB or semiclassical mode wavefunction fails in these cases to approximate the exact wavefunction. The coupled mode semiclassical wavefunction is an accurate approximation of the exact wavefunction over the full range of integration (only part of it is represented). Before the crossing point, it reduces to the single-mode or WKB approximation.

Introducing the $x$-dependent roots of the Hamilton-Jacobi equation (10), $p_{1}, \ldots, p_{4}$ and assuming $p=p_{i}, S_{1}^{\prime}$ can be rewritten as [24]:

$$
S_{1}^{\prime}=\mathrm{i} \sum_{j \neq i} \frac{1}{p_{i}-p_{j}} \frac{\mathrm{d} p_{i}}{\mathrm{~d} x}
$$

Thus, we readily realize that near degeneracy makes $S_{1}^{\prime}$ diverging whereas $S_{0}^{\prime}$ remains constant, thus breaking condition (14).

The conclusion that can be made so far is that near degeneracy of the momenta given by (10) is correlated to the failure of the semiclassical approximation and to the occurrence of probability transfer. It leads us to give up the attempt to describe probability transfer by means of a WKB expansion. 


\section{New semiclassical expansion and probability transfer}

\subsection{Coupled-mode semiclassical expansion}

Let us focus on two-level wave equation (8) and rewrite it first under the general following form:

$$
\left(\frac{\mathrm{d}^{4}}{\mathrm{~d} x^{4}}-\mathrm{i} a_{1}(x) \frac{\mathrm{d}^{3}}{\mathrm{~d} x^{3}}-a_{2}(x) \frac{\mathrm{d}^{2}}{\mathrm{~d} x^{2}}+\mathrm{i} a_{3}(x) \frac{\mathrm{d}}{\mathrm{d} x}+a_{4}(x)\right) \alpha(x)=0 .
$$

As (8) is a real coefficient differential equation, $a_{1}$ and $a_{3}$ are imaginary coefficients. Its associated Hamilton-Jacobi equation is:

$$
J_{4}(x, p)=p^{4}-a_{1}(x) p^{3}+a_{2}(x) p^{2}-a_{3}(x) p+a_{4}(x)=0 .
$$

The $a_{j}(x)$ coefficients appear to be the $j$ th-order sum of the roots of (17). As (16) is derived from a real Schrödinger equation, (17) is a real coefficient equation with respect to the variable i $p$. Then if $\mathrm{i} p$ is a solution, its conjugate $(\mathrm{i} p)^{*}$ is too. In the following, we assume that:

$p_{3}=-p_{1}^{*} \quad p_{4}=-p_{2}^{*} \quad$ and $\quad \Re\left(p_{1}\right)>0 \quad \Re\left(p_{2}\right)>0$.

In order to develop an alternative semiclassical formalism, let us first study the usual semiclassical approximation, in the optical domain, for simplicity. The differential of the optical path $L$ at the limit of the geometrical optics is:

$$
\mathrm{d} L=n \mathrm{~d} s
$$

where $n$ is the space-dependent refractive index and $\mathrm{d} s$ an infinitesimal distance. This formula tells us that, at the limit of geometrical optics, the wave propagates as if $n$ was locally uniform. This can be easily understood from a physical point of view: the geometrical optics corresponds to the small wavelength limit so that $n$ varies very slowly on a length scale of a few wavelengths. Therefore, the wave 'feels' the propagative medium as uniform. In order to make this remark work in a way other than that leading to the WKB expansion, we then rewrite the wave equation (16) as four first-order coupled equations:

$$
\frac{\mathrm{d}}{\mathrm{d} x}\left(\begin{array}{c}
\alpha_{1} \\
\alpha_{2} \\
\alpha_{3} \\
\alpha_{4}
\end{array}\right)=\left(\begin{array}{cccc}
0 & 1 & 0 & 0 \\
0 & 0 & 1 & 0 \\
0 & 0 & 0 & 1 \\
-a_{4}(x) & -\mathrm{i} a_{3}(x) & a_{2}(x) & \mathrm{i} a_{1}(x)
\end{array}\right)\left(\begin{array}{c}
\alpha_{1} \\
\alpha_{2} \\
\alpha_{3} \\
\alpha_{4}
\end{array}\right)
$$

where $\alpha_{1}=\alpha$, and treat this system in the same way as if the coefficients of the $4 \times 4$ matrix-referred to as $A$-were constants. $A$ in then diagonalized, the resulting matrix is $D$, the transformation matrix is denoted $P$. The new coordinates are denoted $\chi_{k}$ such that: $\alpha_{j}=P_{j k} \chi_{k}$. Straightforward calculations show that $D$ and $P$ depend only on the momenta associated with the Hamilton-Jacobi equation (17) derived from the original wave equation (16). The transformation matrix $P$ defining the new coordinates $\chi_{k}$ is of Van-derMonde type built from the $\mathrm{i} p_{j}$ :

$$
D_{j k}=\mathrm{i} p_{j} \delta_{j k} \quad P_{j k}=\left(\mathrm{i} p_{k}\right)^{j-1}
$$

where $\delta_{j k}$ is Kronecker's symbol. In particular $\alpha$ is simply the sum of the new coordinates $\chi_{j}$.

The original wave equation (16) is then rewritten as a function of the new coordinates $\chi_{j}$ and the four momenta:

$$
\frac{\mathrm{d}}{\mathrm{d} x}\left(\begin{array}{l}
\chi_{1} \\
\chi_{2} \\
\chi_{3} \\
\chi_{4}
\end{array}\right)=\left[\left(\begin{array}{cccc}
\mathrm{i} p_{1} & 0 & 0 & 0 \\
0 & \mathrm{i} p_{2} & 0 & 0 \\
0 & 0 & \mathrm{i} p_{3} & 0 \\
0 & 0 & 0 & \mathrm{i} p_{4}
\end{array}\right)-P^{-1} \frac{\mathrm{d} P}{\mathrm{~d} x}\right]\left(\begin{array}{l}
\chi_{1} \\
\chi_{2} \\
\chi_{3} \\
\chi_{4}
\end{array}\right) .
$$


The matrices $P^{-1}$ and $P^{-1} \mathrm{~d} P / \mathrm{d} x$ are calculated. By means of the relations between $p_{j}$ and $a_{j}$, it is possible to express the matrix element by means of the Hamilton-Jacobi function (17). For the diagonal and off-diagonal terms, noting $v=\partial J_{4} / \partial p$, we get:

$$
\begin{aligned}
& \left(P^{-1} \frac{\mathrm{d} P}{\mathrm{~d} x}\right)_{j j}=\left(\frac{\partial v}{\partial p}\right)_{j} \frac{p_{j}^{\prime}}{2 v_{j}} \\
& \left(P^{-1} \frac{\mathrm{d} P}{\mathrm{~d} x}\right)_{j k}=\frac{p_{j}^{\prime}}{p_{j}-p_{k}} \frac{v_{j}}{v_{k}}
\end{aligned}
$$

where $v=\partial J_{4} / \partial p$ and index $j$ denotes a substitution of $p$ by $p_{j}$ in the resulting function of $p$.

In the full classical limit case of constant coefficients $a_{j}$, the $P^{-1} \mathrm{~d} P / \mathrm{d} x$ term, arising from noncommutation of the operators $\mathrm{d} / \mathrm{d} x$ and $A$, would vanish. We are then led to interpret it as the quantum part of the wave equation, the smaller the regime is the more semiclassical it is. If it is neglected: $\left|P^{-1} \mathrm{~d} P / \mathrm{d} x\right| \ll p_{j}$, the solutions readily obtained:

$$
\exp \left(\mathrm{i} \int p_{j} \mathrm{~d} \xi\right) \quad j=1, \ldots, 4
$$

are the classical wavefunctions (12), restricted to the order zero. Moreover, the expressions (22) and (23) confirm this point of view since $P^{-1} \mathrm{~d} P / \mathrm{d} x$ is globally proportional to the spatial derivative of the momenta: the slower the $a_{j}$, then the $p_{j}$, vary, the smaller the $p_{j}^{\prime}$ will be, hence the matrix $P^{-1} \mathrm{~d} P / \mathrm{d} x$. Writing the wave equation (16) under the form (21) thus allows us to separate in a way the classical and the nonclassical content of (16). In the semiclassical regime, we are then led to treat perturbatively the nonclassical term $P^{-1} \mathrm{~d} P / \mathrm{d} x$. This treatment associated with the rewriting of the wave equation under the form (21) is the heart of the alternative semiclassical procedure we propose.

Let us first neglect the off-diagonal terms of the matrix $P^{-1} A P=D-P^{-1} \mathrm{~d} P / \mathrm{d} x$, thus retaining elements of $D$ and terms of (22). It leads to approximate solutions proportional to the following functions denoted $\chi_{j}^{0}$ :

$\chi_{j}^{0}(x)=\mathrm{e}^{\mathrm{i} \varphi_{j}}=\exp \left(\mathrm{i} \int^{x} p_{j} \mathrm{~d} \xi-\int^{x}\left(\frac{\partial v}{\partial p}\right)_{j} \frac{p_{j}^{\prime}}{2 v_{j}} \mathrm{~d} \xi\right) \quad j=1, \ldots, 4$.

They are readily recognized as the WKB semiclassical expressions of the wavefunctions (12) up to first order. As they are obtained thanks to a diagonal matrix (off-diagonal terms set to zero), $\chi_{j}^{0}$ are denoted the semiclassical modes associated with the wave equation (16). The wave component $\alpha$ is then a linear combination of these modes. The change of basis by means of the matrix $P$ thus allows one to recast the initial wave equation (16) under the form (21) where the WKB wavefunctions appear naturally, avoiding resorting to any expansion whatsoever. The effect of the off-diagonal terms is then to couple the semiclassical modes. The weak-coupling condition is fulfilled provided that the off-diagonal terms of the matrix $P^{-1} \mathrm{~d} P / \mathrm{d} x$ can be neglected with respect to the diagonal matrix $D$. It requires that:

(i) The momenta $p_{j}$ or the coefficients of the wave equation (16) vary slowly (small derivatives) which corresponds to the usual condition of slow variation of the de Broglie wavelength in the classical regime. This ensures that $\mathrm{d} P / \mathrm{d} x$ is small.

(ii) The momenta solutions of (17) must be sufficiently separated in order to avoid quasi-vanishing denominators in (23).

Note that, when conditions (i) and (ii) are satisfied, the diagonal part of $P^{-1} \mathrm{~d} P / \mathrm{d} x$ should also be negligible with respect to the momenta $p_{j}$. Nevertheless, it is not neglected 
in the expression of $\chi_{j}^{0}$ because the momentum $p_{j}$, which is mainly real here, gives in $\chi_{j}^{0}$ a nearly pure phase (imaginary) term whereas $(\partial v / \partial p)_{j} p_{j}^{\prime} / 2 v_{j}$ gives essentially an amplitude (real) term. Hence, whatever the magnitude of the momentum, this term has a noticeable effect on the wavefunction and is thus retained in the expression (24).

Another condition should be added, concerning the generalized speed $v_{j}$, which is to avoid the vicinity of turning points $\left(v_{j}=0\right)$. As our treatment of nonadiabatic coupling is semiclassical, we assume that no turning points are located in the vicinity of an avoidedcrossing region.

Similar conditions were already noted in writing the convergence condition of the WKB expansion of the solutions of (8), that is inequality (14) and equation (15). The previous formalism puts these requirements in a new light, it provides a validation of the WKB expansion in cases satisfying conditions (i) and (ii).

Historically, approximations (12) or (24) were already derived prior to Wentzel, Kramers and Brillouin (1926) by Liouville and Green (1837) [25] thanks to a method not equivalent to an expansion with respect to a small parameter. The method stated before is a new way of deriving this result that should rather be called Liouville-Green approximation. The contribution of the first authors concerns the connection formula through a turning point. However, in order not to change common use, we will give the name WKB approximation to the semiclassical modes (24).

We return now to the original problem of nonadiabatic transitions. As noted, the WKB wavefunctions or the semiclassical modes (24) are accurate approximations of the exact wavefunction in the case where no transfer occurs. Consequently, in the frame of our formalism, the nonadiabatic transfer will be understood as semiclassical mode conversion. It means that the semiclassical modes are no longer valid approximations of the exact wavefunction, the neglected off-diagonal terms (23) shall be taken into account because condition (ii) is no longer fulfilled. Quasi-degeneracies of the momenta appear which correspond, as noted, to the breakdown of the semiclassical approximation.

Before computing the wavefunction, let us make a useful simplification of (21). In this regime, momenta have large absolute values: from property (18), the momenta $p_{1}$ and $p_{2}$ are well separated from $p_{3}$ and $p_{4}$ respectively. From condition (ii) we can conclude that no semiclassical mode conversion occurs between the pairs of modes $(1,2)$ and $(3,4)$. If modes 1 and 2 are chosen so that their real part is positive, they represent the progressive semiclassical waves whereas modes 3 and 4 are the counter-progressive ones. The previous statement is thus simply the fact that, in the classical regime, no mode conversion occurs between modes of opposite direction. It allows us to neglect coupling terms $\left(\left(P^{-1} \mathrm{~d} P / \mathrm{d} x\right)_{j k}\right.$, $j=1,2, k=3,4)$ between these modes, that is, to derive two equations coupling two modes. For modes 1 and 2, (21) is approximated to:

$$
\begin{aligned}
& \frac{\mathrm{d} \chi_{1}}{\mathrm{~d} x}=\left(\mathrm{i} p_{1}-\left(\frac{\partial v}{\partial p}\right)_{1} \frac{p_{1}^{\prime}}{2 v_{1}}\right) \chi_{1}-\frac{p_{2}^{\prime}}{p_{1}-p_{2}} \frac{v_{2}}{v_{1}} \chi_{2} \\
& \frac{\mathrm{d} \chi_{2}}{\mathrm{~d} x}=\left(\mathrm{i} p_{2}-\left(\frac{\partial v}{\partial p}\right)_{2} \frac{p_{2}^{\prime}}{2 v_{2}}\right) \chi_{2}-\frac{p_{1}^{\prime}}{p_{2}-p_{1}} \frac{v_{1}}{v_{2}} \chi_{1} .
\end{aligned}
$$

Thus, the first result of the coupled mode formalism is to reduce the initial fourth-order Schrödinger equation (16) to a set of two coupled first-order ones.

The coupling region of the modes corresponds to the vicinity of the crossing point where condition (ii) is violated. Let us assume that only $\chi_{j}, j=1$ or 2 , is different from zero far from the crossing, that is $\alpha=\chi_{j}=\chi_{j}^{0}$. We apply an iterative scheme of resolution of (25) and (26) considering the off-diagonal terms of $P^{-1} \mathrm{~d} P / \mathrm{d} x$ as perturbing terms of the semiclassical mode $j$, taken naturally as an initial value of the iteration. It provides the 
coupled modes semiclassical wavefunction. Up to order $2 n$, it reads:

$\alpha_{(j)}(x)=\left(1+K_{2}(x)+\cdots+K_{2 n}(x)\right) \chi_{j}^{0}(x)+\left(K_{1}(x)+\cdots+K_{2 n-1}(x)\right) \chi_{k}^{0}(x)$

where

$$
\begin{aligned}
& K_{1}(x)=\int_{x_{0}}^{x}-\frac{p_{j}^{\prime}}{p_{k}-p_{j}} \frac{v_{j}}{v_{k}} \mathrm{e}^{\mathrm{i}\left(\varphi_{j}-\varphi_{k}\right)} \mathrm{d} \xi \\
& K_{2}(x)=\int_{x_{0}}^{x}-\frac{p_{k}^{\prime}}{p_{j}-p_{k}} \frac{v_{k}}{v_{j}} K_{1} \mathrm{e}^{\mathrm{i}\left(\varphi_{k}-\varphi_{j}\right)} \mathrm{d} \xi \\
& \vdots \\
& K_{2 n+1}(x)=\int_{x_{0}}^{x}-\frac{p_{j}^{\prime}}{p_{k}-p_{j}} \frac{v_{j}}{v_{k}} K_{2 n} \mathrm{e}^{\mathrm{i}\left(\varphi_{j}-\varphi_{k}\right)} \mathrm{d} \xi \\
& K_{2 n+2}(x)=\int_{x_{0}}^{x}-\frac{p_{k}^{\prime}}{p_{j}-p_{k}} \frac{v_{k}}{v_{j}} K_{2 n+1} \mathrm{e}^{\mathrm{i}\left(\varphi_{k}-\varphi_{j}\right)} \mathrm{d} \xi .
\end{aligned}
$$

$x_{0}$ is the starting point of integration in an uncoupled region. The index $(j)$ of $\alpha$ is here to recall that only $\chi_{j}^{0}$ is populated at $x_{0}, k$ being the other mode (not to be confused with indexes in (19)-(24)).

The phenomenon quantified by (28)-(31), will be called semiclassical mode conversion between $\chi_{j}^{0}(x)$ and $\chi_{k}^{0}(x) . K_{1}$ is the first-order term of conversion between the initial mode and the other one. Examination of expressions (28)-(31) clearly shows that this conversion is appreciable when, first, the usual classical condition is violated, that is, when rapid variations or low values of the impulsion are encountered ( $p_{1,2}^{\prime}$ large or phases $\varphi_{1,2}$ slowly varying) and, second, in the vicinity of a near degeneracy of the impulsions $\left(p_{1}-p_{2}\right.$ approaching zero).

As the initial wave equation (6) is under spinorial form, we shall derive the second component $\beta(x)$ from $\alpha(x)$. By means of the first line of (6), it leads simply to:

$$
\beta(x)=\frac{1}{V}\left(\frac{1}{2 m} \frac{\mathrm{d}^{2}}{\mathrm{~d} x^{2}} \alpha(x)+\left(E-V_{1}(x)\right) \alpha(x)\right) .
$$

Rewriting the wave equation under the form (21) makes the calculation of the second-order derivative easy. In (19), $\alpha_{3}$ is the second-order derivative of $\alpha$, so that from passage matrix $P$, we obtain:

$$
\alpha(x)^{\prime \prime}=\sum_{j=1}^{4}-p_{j}^{2} \chi_{j}
$$

Hence, the total wavefunction can be derived from the $\chi_{j}$ with no explicit spatial derivative calculation which simplifies numerical computations. In the regime where the semiclassical modes are uncoupled-one mode can be propagated while the others remain with their amplitude equal to zero-the sum in the previous equation reduces to one term. Then, thanks to (32), one can define the $\beta$-component $\kappa_{j}^{0}$ corresponding to the mode $\chi_{j}^{0}$ :

$$
\kappa_{j}^{0}(x)=\frac{-p_{j}^{2}(x) / 2 m+E-V_{1}(x)}{V} \chi_{j}^{0}(x) .
$$

Numerical validation of that formalism has been made in the previously studied situations of section 2.2. In the first of these cases, a low value of the diabatic coupling $V$ and a low value of the energy $E$ has been chosen, the order of iteration required is 6 . The corresponding result is reported in figure 2(a). The study of the case of large $V$ and $E$ is reported in figure $2(b)$; the same order is required. These numerical computations 
validate then both the approximation leading to the reduced set (25) and (26) and the accuracy achieved by the iterative scheme. The coupled mode semiclassical formalism thus allows us to interpret the nonadiabatic coupling at the semiclassical limit where the WKB approximation fails. It leads to the iterative scheme (28)-(31).

From the beginning, we supposed that the diabatic coupling potential $V$ was a constant, in order to simplify expression (8). It is now obvious that this is not a limiting assumption as far as this parameter is never equal to zero. The elimination of the $\beta$-component of the wavefunction from (6) can be done, leading to a general fourth-order differential equation (16), the starting point of the study.

From numerical computations, we conclude that such a coupled mode semiclassical formalism allows us to deal, in the classical regime, with wave equations whose momenta are nearly degenerate. It gives a deeper understanding of the semiclassical limit as a broader class of wave equations are taken into account. The procedure, beginning with the rewriting of the wave equation under the form (19) and leading to the iterative scheme of (28)-(31), constitutes a semiclassical expansion fundamentally distinct from the WKB one. It gives a rather natural derivation of the first-order WKB term and probably explains why, at this order, the WKB wavefunction is an accurate approximation (under conditions (i) and (ii)), but subsequently the coupled mode and the WKB expansion are different. Whereas the WKB expansion is of asymptotic nature and immediately diverges when appreciable probability transfer occurs, numerical computations show that the coupled semiclassical modes scheme practically achieves to give the wavefunction very accurately in such a situation. Thus, this new formalism advantageously replaces the standard WKB expansion. The general study of the convergence of the iterative expansion will not be carried out here.

\section{Applications}

Let us now give three applications of this theory: the first one dealing with ordinary (scalar) Hamiltonians, the following two dealing with two-level Hamiltonians. The first of them concerns transition probability compared with the LZ formula, the second concerns the evolution of wavepackets.

\subsection{Scalar Hamiltonian}

The preceding theory can be successfully used to recover an already known result about the reflection of a wavepacket above a potential barrier. A wavepacket whose energy is entirely above a potential barrier undergoes a partial reflection if its average energy is near the top of the barrier. From a time-independent point of view, it means that a progressive wave far away on one side of the barrier is connected to the progressive and counterprogressive waves on the other side [26]. Such property clearly prevents a WKB expansion converging to the exact solution as it cannot contain both opposite waves. Classically, the accessible phase space exhibits a near degeneracy point corresponding to the top of the barrier. We may apply our previous theory: the point of near degeneracy is regarded as a semiclassical mode conversion point between the semiclassical modes corresponding to the progressive and counter-progressive waves far from the barrier. The classical Hamiltonian or the Hamilton-Jacobi function is chosen as:

$$
H=\frac{p^{2}}{2 m}+V(x) \quad \text { where } V(x)=5 \mathrm{e}^{-x^{2}}
$$


the momenta are then:

$$
p_{+}=p=\sqrt{2 m(E-V(x))} \quad \text { and } \quad p_{-}=-p
$$

where $E$ is the energy. The corresponding time-independent Schrödinger equation is readily derived from Hamiltonian (35). The procedure of diagonalization of the previous subsection allows us to rewrite it, with obvious notation for the new coordinates, under the following form:

$$
\frac{\mathrm{d}}{\mathrm{d} x}\left(\begin{array}{c}
\chi_{+} \\
\chi_{-}
\end{array}\right)=\left(\begin{array}{cc}
\mathrm{i} p-p^{\prime} / 2 p & p^{\prime} / 2 p \\
p^{\prime} / 2 p & -\mathrm{i} p-p^{\prime} / 2 p
\end{array}\right)\left(\begin{array}{c}
\chi_{+} \\
\chi_{-}
\end{array}\right) .
$$

From the diagonal part of the preceding matrix, the classical modes are readily recognized as the usual WKB progressive and counter-progressive waves.

As before, setting $E=5.7$ near the top of the barrier, we made a numerical comparison between the exact wavefunction, the WKB wavefunction (12) and the mode coupled semiclassical wavefunction, retaining only the two first orders, which are displayed in figure 3. The integration proceeds from negative $x$ (i.e. only mode minus is populated for $x \ll 0$ ), and this corresponds to a wavepacket coming from positive $x$ and partially reflected towards positive $x$ and transmitted towards negative $x$ at the encounter with the barrier. As $E$ tends towards the top of the barrier, the WKB wavefunction fails to fit the exact one in the $x>0$ region. For values of $E$ not too close to the top of the barrier, the second-order wavefunction successfully fits.

More particularly, (28) gives, to first order, the reflection coefficient $K_{r}$ of a single wave of energy $E$. Limits of integration are extended to $-\infty$ and $+\infty$ as the integrand contributes notably only in the vicinity at the top of the barrier. It leads to the formula:

$$
K_{r}=\int_{-\infty}^{+\infty} \frac{p^{\prime}}{2 p} \mathrm{e}^{\mathrm{i} \int_{-\infty}^{x} 2 p \mathrm{~d} x^{\prime}} \mathrm{d} x .
$$

It is exactly the one found by Bremmer, see references and discussions in [22]. Bremmer's original method consists of replacing the potential by a staircase function, solving exactly the Schrödinger equation by applying a connection formula around vertical steps and, finally, letting the dimension of each stair go to zero. The approach followed here clearly shows the physical origin of the reflected wave in the impossibility of neglecting the off-diagonal terms

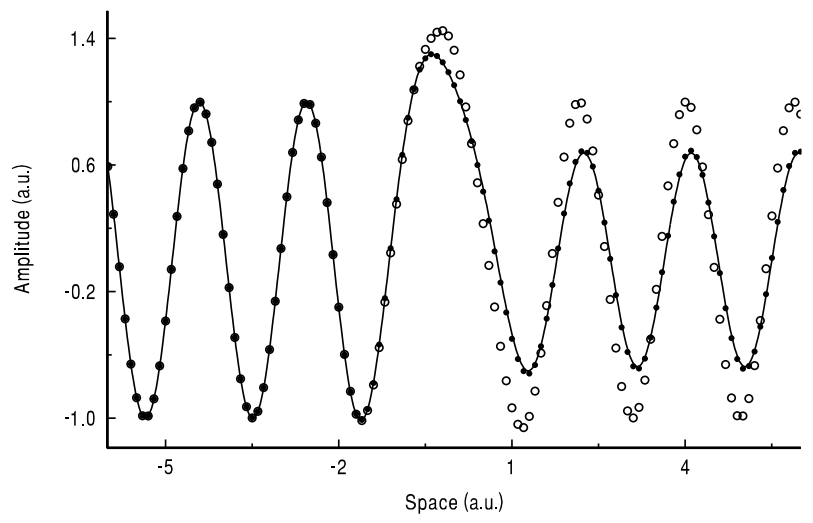

Figure 3. Reflection above the potential barrier $V(x)=5 \mathrm{e}^{-x^{2}}$ with $E=5.7$. Symbols as in figure 2. A change of amplitude and phase is encountered around the top of the barrier $(x=0)$. Before this point, all three wavefunctions coincide; after, only the coupled mode wavefunction achieves to fit the exact one. 
of the matrix $P^{-1} \mathrm{~d} P / \mathrm{d} x$ in the vicinity of the top of the barrier. The field of application of the WKB technique is clearly circumvented here. An approach [27] other than Bremmer's, consists of writing the wavefunction as an $x$-dependent linear combination of the WKB zeroth- and first-order term wavefunctions. It leads to the same iterative scheme as ours. The recasting of the Schrödinger equation under the form (36) is, we think, a more natural starting point.

\subsection{Transition probability}

We consider the adiabatic potentials of figure 4(a) simply made of tangent hyperbolic functions coupled by a flat potential of value 1.5. Such a choice was made for two reasons: it allows us to deal with constant slope diabatic potentials around the crossing point coupled by a constant in order to be in 'standard' conditions where the LZ formula should work. Secondly, in the constant potential regions, both the adiabatic and the singlemode approximations are valid. The correspondence between these wavefunctions is then easy, as well as the calculation of asymptotic momenta. In the uncoupled regions, the
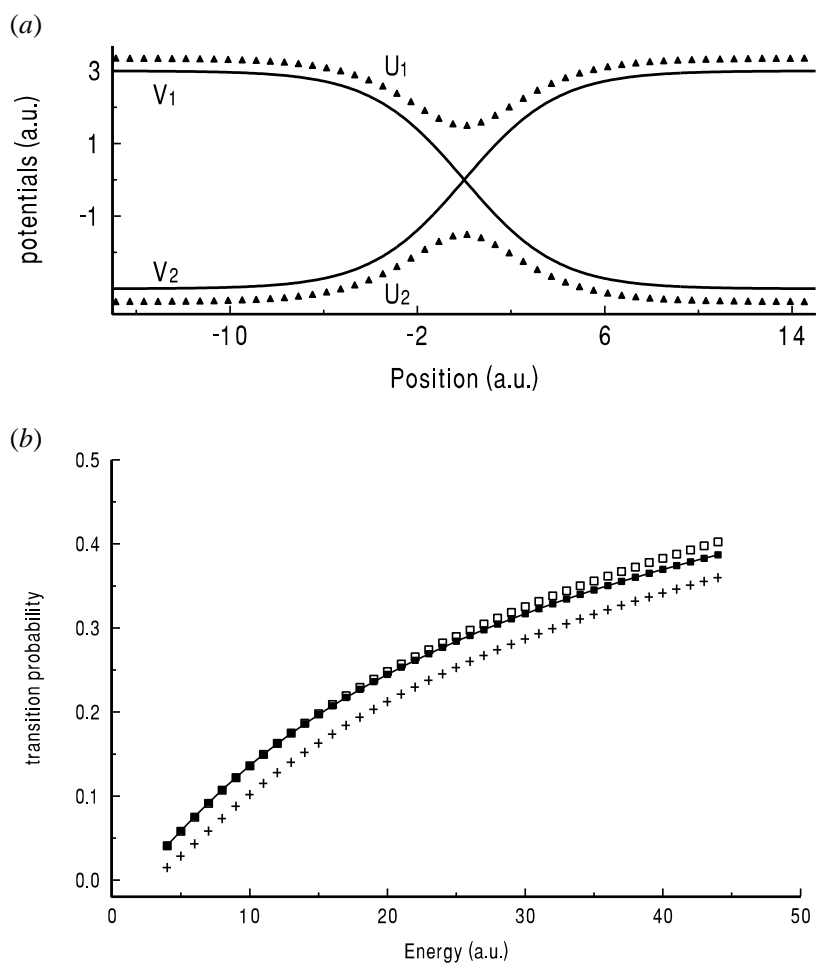

Figure 4. (a) represents the potentials (diabatic and adiabatic) chosen for the study. The full curves are the diabatic potentials $V_{1}$ and $V_{2}$. The scattered curves are the adiabatic potentials $U_{1}$ and $U_{2}, V=1.5$. In $(b)$ are compared four quantities: the exact probability transfer (full curve), the formula from the coupled mode formalism reduced to the first order ( $\square$ ) and to the third order $(\mathbf{\square})$, and the LZ formula (+). Despite the straight line shape of the diabatic potentials at the crossing point, the LZ formula fails to predict the nonadiabatic transition probability whereas the use of the coupled mode formalism succeeds at a small order of iteration. 
diabatic wavefunction is, in general, a linear combination of modes 1 and 2, then

$$
\left(\begin{array}{c}
\alpha \\
\beta
\end{array}\right)=A_{1}\left(\begin{array}{l}
\chi_{1}^{0} \\
\kappa_{1}^{0}
\end{array}\right)+A_{2}\left(\begin{array}{l}
\chi_{2}^{0} \\
\kappa_{2}^{0}
\end{array}\right) \text {. }
$$

If one denotes $\left(\begin{array}{c}\breve{\alpha} \\ \vec{\beta}\end{array}\right)$ as the corresponding adiabatic wavefunction, the diabatic adiabatic basis transformation leads to the relations:

$$
\begin{aligned}
& \breve{\alpha}=A_{1} \frac{\chi_{1}^{0}}{\cos (\theta / 2)} \\
& \breve{\beta}=A_{2} \frac{\chi_{2}^{0}}{\sin (\theta / 2)}
\end{aligned}
$$

where $\theta$ is the angle satisfying:

$$
\tan (\theta)=\frac{2 V}{V_{1}-V_{2}} .
$$

The calculation is not given in detail, it is based on the fact that in the regions of constant potentials, $\chi_{j}^{0} \propto \mathrm{e}^{\mathrm{i} \breve{p}_{j} x}$ where $\breve{p}_{j}$ satisfies $\breve{p}_{j}^{2} / 2 m+U_{j}=E$. The incoming region is left of the crossing point $(x<0)$, only mode 1 is populated here which is equivalent to populating the surface $U_{1}$. The outgoing region is right of the crossing point $(x>0)$ where both surfaces are populated. The nonadiabatic probability transfer is then:

$$
\operatorname{Pr}=\frac{|\breve{\beta}(+\infty)|^{2}}{|\breve{\alpha}(-\infty)|^{2}} \frac{\breve{p}_{2}(\infty)}{\breve{p}_{1}(\infty)}
$$

The notation $\pm \infty$ corresponds, respectively, to the $x<0$ and the $x>0$ asymptotic regions where the potentials are constant. The difference of momenta between the two surfaces, in the asymptotic region, induces a difference of flux that is included in the above formula. In terms of semiclassical modes, it reads, thanks to relations (39) and (40):

$$
\operatorname{Pr}=\left|1+K_{1}(+\infty)+K_{3}(+\infty)+\cdots\right|^{2}\left|\chi_{2}^{0}(+\infty)\right|^{2} \sqrt{\frac{E-U_{2}}{E-U_{1}}}
$$

assuming that mode 1 is populated initially such that $\left|\chi_{1}^{0}(-\infty)\right|=1$. Let us note that $\theta(+\infty)=\pi-\theta(-\infty)$ which explains why $\theta$ does not appear in the formula. The LZ formula [5] reads:

$$
\operatorname{Pr}=\exp \left(-\frac{2 \pi V^{2}}{\sqrt{2(E-V)}\left(s_{1}-s_{2}\right)}\right)
$$

where $V$ is the diabatic coupling, $E$ the energy and $s_{1}$ and $s_{2}$ are the slopes of the potentials at the crossing point $(\hbar=1)$. In Child [7], the square root is simply $\sqrt{2 E}$. The numerical study is made as a function of the energy $E$, the results are reported in figure $4(b)$. In that configuration of potentials, for the range of energy considered, the LZ formula constantly underestimates the probability transfer by about 0.03 . This makes the largest relative error at low energies. In contrast, the value predicted by the coupled mode formalism reduced to the first-order term $K_{1}$ is good, at least at low energies. When the third-order term is included, we can see the convergence of the iteration scheme. Even when only the first-order term is taken into account, computation of probability $P$ involves non-trivial calculations of the integrals (28)-(31), even in an approximate way. Let us notice that in the case considered here (figure 4(a)), the difference of slope of the diabatic potential at the crossing point leads to a rather localized interaction region so that a small order of iteration is required, the region contributing to the necessary integrals being restricted to the vicinity of this point. 


\subsection{Generalized group velocity}

Until now, our semiclassical study of coupled modes was restricted to time-independent wave equations. We will show in this section how this formalism can be used to derive the motion of wavepackets in a two-level system. Let us first recall the notion of group velocity and explain how it can be generalized. In a uniform media or in a constant potential, a scalar wave has a spacetime dependence equal to $\exp [\mathrm{i}(k x-\omega t)]$. The relation between the wave vector $k$ and the pulsation $\omega$-resulting from the wave equation-is the dispersion relation $\mathcal{D}(k, \omega)=0$. The phase velocity $v_{\varphi}$ is then simply the ratio $\omega / k$. But the velocity of a wavepacket, of mean wave vector $k_{0}$, is given by the so-called group velocity $v_{g r}$ :

$$
v_{g r}=\left(\frac{\mathrm{d} \omega}{\mathrm{d} k}\right)_{k=k_{0}}=-\frac{\partial \mathcal{D} / \partial k}{\partial \mathcal{D} / \partial \omega} .
$$

The first equality is obtained thanks to the stationary phase approximation applied to the wavepacket expression written as a sum of the previously mentioned elementary waves. The second equality is obtained by a mere differentiation of the dispersion relation. As we can see, a stationary analysis of a wave equation allows us to derive the law of evolution of wavepackets. Our aim is then to generalize this notion to any wave equation.

The first extension of the group velocity can be applied to a general wave equation at the usual (single mode) semiclassical limit. In that regime, the wavefunctions are given by the WKB form, as shown before, i.e.

$$
A(E, x) \exp \left(\mathrm{i}\left[\int^{x} p(E, \xi) \mathrm{d} \xi-E t\right]\right) .
$$

The $A(E, x)$ amplitude factor, corresponding to the first-order term in the WKB expansion, is slowly varying on the scale of the spatial phase $1 / p$ if the condition (14) holds. $p, E$ and $x$ are related through the Hamilton-Jacobi equation. A space-localized wavepacket can be expressed as a sum over the energy of the elementary waves (45):

$$
\psi(x, t)=\int a(E) A(E, x) \mathrm{e}^{\left(\mathrm{i}\left[\int_{0}^{x} p(E, \xi) \mathrm{d} \xi-E t\right]\right)} \mathrm{d} E .
$$

$a(E)$ is the projection of the wavepacket $\psi(x, 0)$ on the wave $(45)$ at time $t=0, \psi(x, 0)$ is assumed to be spatially centred around 0 and energetically around a given value $E_{0}$. Under the assumption of a sufficiently localized wavepacket compared with the typical length of variation of $p$-which is of the same order as the typical length of variation of the potentials - we expand $p$ with respect to space in expression (46) around 0 , then $p(E, x)=p(E, 0)+p^{\prime}(E, 0) x+\cdots$. The classical regime hypothesis allows us to make sense of such an expansion as $p^{\prime}$ is assumed to be small. Retaining the zeroth-order term, it gives a space- and time-localized expression of the wavepacket around $x=0$ and $t=0$ as a sum of waves of the pattern $\exp [\mathrm{i}(p(E, 0) x-E t)]$ already studied. Applying the previous result, we can derive the speed $v_{g r}$ of this wavepacket locally, i.e.

$$
v_{g r}=\left(\frac{\partial E(p, x)}{\partial p}\right)_{E=E_{0}} .
$$

The preceding argument can be repeated at each point, hence the expression (47) is the velocity of the wavepacket everywhere. If $J$ is the Hamilton-Jacobi equation connecting the variables $E, p, x$, then a mere differentiation gives:

$$
v_{g r}=\frac{-\partial J / \partial p}{\partial J / \partial E}
$$


This expression, compared with equation (44), confirms in a way the fact that the HamiltonJacobi equation is a generalized dispersion relation.

We have here, for simplicity, implicitly assumed that the Hamilton-Jacobi equation derived in the classical regime was real. The case of a complex equation will be examined later.

The second extension will concern the coupled mode semiclassical wavefunctions. The elementary wave is rewritten under the form of the sum of two coupled semiclassical modes:

$$
\begin{aligned}
\psi(x, t) & =\chi_{1}(E, x) \mathrm{e}^{-\mathrm{i} E t}+\chi_{2}(E, x) \mathrm{e}^{-\mathrm{i} E t} \\
& =\varrho_{1}(E, x) \mathrm{e}^{\mathrm{i} \int \mathcal{S}_{1}^{\prime}(E, \xi) \mathrm{d} \xi-\mathrm{i} E t}+\varrho_{2}(E, x) \mathrm{e}^{\mathrm{i} \int \mathcal{S}_{2}^{\prime}(E, \xi) \mathrm{d} \xi-\mathrm{i} E t}
\end{aligned}
$$

where $\varrho_{j}$ and $\mathcal{S}_{j}$ are real. Note that the $K_{n}$ factors are then involved in both of these. The extension of the previous theory requires that the moduli $\varrho_{j}$ vary slowly on the length scale of $1 / \mathcal{S}_{j}$ which is not obvious. We shall not discuss this fact from the theory of coupled mode but simply give arguments from numerical computations. The example studied uses potentials of figure 4(a) at energy $E=15$, mode 1 is the initially populated mode. Numerically, the derivatives of the phases $\mathcal{S}_{j}{ }_{j}$ remain of the order of the momenta $p_{j}$, that is, a few units. Besides, the maximum variation of the moduli $\varrho_{j}$ with respect to space is one-tenth, see figure 5(a). The required condition of slow variation of the moduli is thus fulfilled.

Before calculating group velocities, let us add something about the phases. For the incoming mode, $\mathcal{S}^{\prime}{ }_{1}$ is nearly equal to the corresponding momentum $p_{1}$ all over the space. In contrast, for the generated mode, $\mathcal{S}_{2}^{\prime}$ is first nearly equal to $p_{1}$, then it goes progressively to $p_{2}$ as the crossing point is passed. As we can expect, this means that the phase velocities of the two modes are equal when the second is born, only further the phase velocity of the generated mode goes to its asymptotic value. The effect of the $K_{n}$ factors on the phase of $\chi_{2}$ is hence crucial to get this result.

Applying the theory of the preceding paragraph, we can derive two group velocities corresponding to each mode. They are:

$$
v_{g r j}=\left(\frac{\partial \mathcal{S}_{j}^{\prime}(E, x)}{\partial E}\right)^{-1} \text {. }
$$

Let us make three remarks. First, in the case of uncoupled semiclassical modes with a real Hamilton-Jacobi equation, the preceding formula (50) reduces to the already derived one (47). Second, up to the first generalization, real Hamilton-Jacobi equations hence real momenta have been considered. In the case that they are complex, the semiclassical wavefunction shall be written under the previous form (49) and equation (50) shall be applied. Third, this theory is quite rudimentary as no corrections coming from the amplitude of the elementary waves was taken into account. Nevertheless, it gives physically coherent results as we shall see.

The expected overall result of these successive generalizations of the notion of group velocity is to derive a two-wavepacket theory as announced in the introduction, demanded for physical reasons. The derivatives of the phases $\mathcal{S}_{j}^{\prime}$ are easily calculated thanks to the relation:

$$
\frac{\mathrm{d} \chi_{j} / \mathrm{d} x}{\chi_{j}}=\frac{\varrho_{j}^{\prime}}{\varrho_{j}}+\mathrm{i} \mathcal{S}_{j}^{\prime}
$$

and (25), (26). The derivative with respect to energy has to be done numerically. We assume only mode 1 populated in the incoming region $(x<0)$, the group velocity for mode 2 is calculated where its amplitude is sufficient $\left(\left|\chi_{2}\right|>5 \times 10^{-3}\right)$. The result is reported 

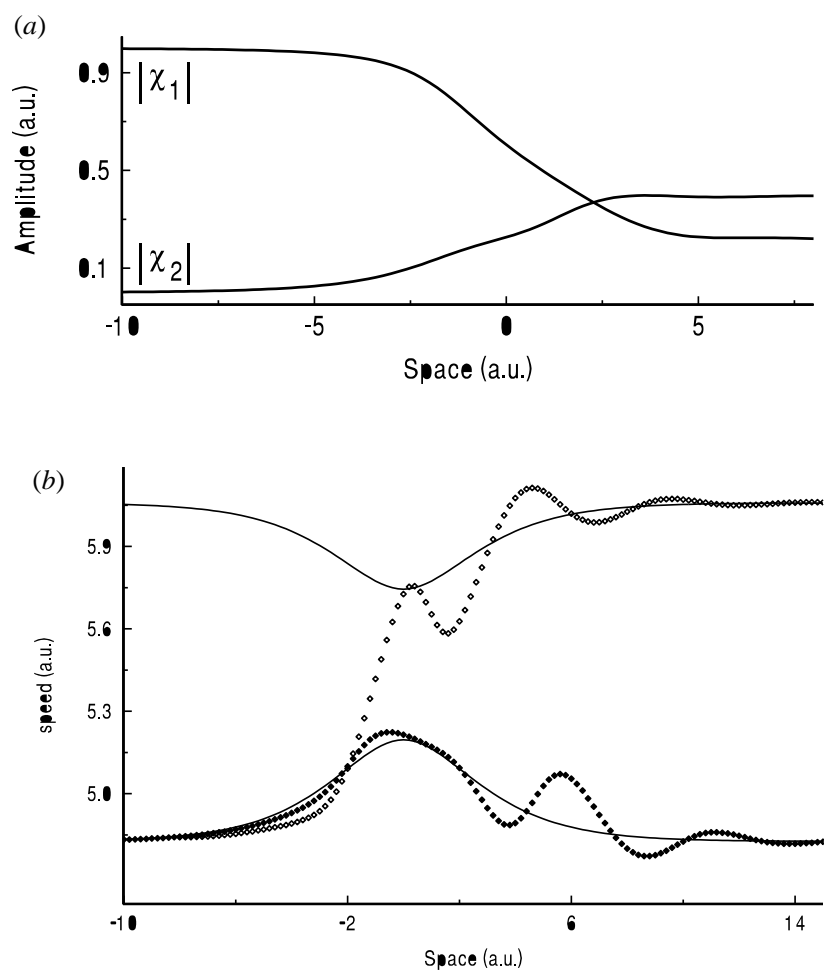

Figure 5. $(a)$ is a plot of the amplitudes of the modes 1 and 2 as a function of space in order to show that they vary smoothly on the scale of $1 / p_{j}$. In $(b)$, the full curves are the adiabatic momenta associated with the adiabatic potentials $U_{1}$ and $U_{2}$ of figure 4(a). The generalized group velocities of the two modes are plotted as a function of space: the mode of the initially populated surface $(\diamond)$ and the generated mode $(\diamond)$. As expected, the wavepackets, initially together, separate at the crossing point and eventually are decoupled in the asymptotic adiabatic region $(x>0)$.

in figure 5(b) where group velocities are plotted as a function of space. A comparison is made between the velocities in the adiabatic approximation. As for the phase velocities, one notes that an equality in region mode 2 is born. In the incoming region, the generated wavepacket of mode 2 first follows the main wave of mode 1 . In the vicinity of the crossing point, these waves separate and, at the end, move independently in the outgoing region with their asymptotic speeds. The corresponding $\beta$-component is of the same shape as $\alpha$, since relation (32) prevents $\beta$ from being different from zero where $\alpha$ is. This does not mean that the wavepackets are centred around the same point, but it prevents a splitting effect similar to those occurring between modes. Thus this computation, besides arguments already stated, confirms the well founded nature of the idea of generalizing group velocity in the case of coupled modes. It allows us to reach the aim, announced in the introduction, to derive a semiclassical formalism not reduced to a single wavepacket assumption.

Let us conclude that in the case of bound states, which was the initial motivation of our study, reflections of wavepackets on the potentials lead to a successive passage on the crossing point so that several splittings occur. The result of the coupled mode semiclassical formalism from a time-dependent point of view, is thus a multiple wavepackets theory. 


\section{Conclusion}

As we have shown, the spinorial or two-level Schrödinger equations can be the object of a semiclassical treatment. The essential different feature from the ordinary one-level Schrödinger equation is that there are two propagating semiclassical waves, obtained through the WKB approach. Whereas in the usual (nonspinorial) case, the limit $E \rightarrow \infty$ separates the momenta of the propagative and counter-propagative semiclassical waves, it leads, in our spinorial case, to the near degeneracy of the momenta of same sign, causing the failure of WKB approximation. The same fact is encountered when the diabatic coupling decreases. In both of these cases, appreciable probability transfer occurs, which is precisely the central fact within which we want the semiclassical approximation to work. We show then that an alternative semiclassical iterative treatment is possible, allowing us to deal with these limiting cases. It leads to the coupled mode semiclassical formalism and clearly shows limits of traditional approaches. The well known example of the reflection of a wavepacket over a potential barrier gives, by analogy, an intuitive idea of what happens in a two-level system.

Let us stress the fact that the coupled mode formalism is indeed of semiclassical nature because, as the WKB formalism, it allows us to integrate the Schrödinger equation by successive quadratures by the unique means of the classical momenta. Its advantage is that it accurately approximates the wavefunction in cases the WKB method does not.

This formalism provides a sort of canonical separation of the wavefunction as a sum of four, rather often two, functions that can be interpreted physically by means of their associated wavepackets. The derivation of the corresponding velocities, through an extension of the group velocity theory, gives a physically coherent result and leads to the expected splitting of the wavepacket at the encounter with the crossing point.

From the practical point of view of calculating wavefunctions, the coupled mode formalism would be interesting in a multidimensional space as it would allow us to deal with ordinary differential equations rather than partial differential equations for which space dimension is a rapidly limiting factor. However, such a theory cannot be generalized straightforwardly to two dimensions as the Laplacian operator involved in the Schrödinger equation cannot be factored into two equal differential operators of first order. Nevertheless, a way of circumventing this difficulty is currently considered by embedding the position space in a larger one.

The second problem addressed in this paper is the derivation of dynamical behaviour of particles in two-level systems. A way to deal with it has been sketched. Improvements can be made by use of the stationary phase techniques. Unlike the previously mentioned problem, the case of multidimensional position space is not a priori a difficulty.

\section{References}

[1] Delon A, Jost R and Lombardi M 1991 J. Chem. Phys. 955701

[2] Bohigas O 1991 Chaos and Quantum Physics (Les Houches 1989, Session LII) (Amsterdam: North-Holland)

[3] Berry M 1985 Proc. R. Soc. A 400229

[4] Bogomolny E, Georgeot B, Giannoni M J and Schmit C 1992 Phys. Rev. Lett. 692188

[5] Zener C 1932 Proc. R. Soc. 137696

[6] Thorson W R, Delos J B and Boorstein S A 1971 Phys. Rev. A 41052

[7] Child M S 1974 Molecular Collision Theory (New York: Academic)

[8] Meyer H D and Miller W H 1979 J. Chem. Phys. 703214

[9] Delos J B 1981 Rev. Mod. Phys. 53287

[10] Halcomb L L and Diestler D J 1986 J. Chem. Phys. 843130 
[11] Boiron M, Lombardi M and Wiesenfeld L 1994 Phys. Rev. A 501409

[12] Deumens E, Diz A, Longo R and Öhrn Y 1994 Rev. Mod. Phys. 66917

[13] Delos J B, Thorson W R and Knudson S R 1972 Phys. Rev. A 6709

[14] Landau L and Lifshitz E 1967 Mécanique Quantique (Moscow: Mir) ch VII

[15] Littlejohn R G and Flynn W G 1991 Phys. Rev. A 445239

Littlejohn R G and Flynn W G 1992 Chaos 2149

[16] Frisk H and Guhr T 1993 Ann. Phys. 221229

[17] Littlejohn R G and Flynn W G 1994 Ann. Phys. 234334

[18] Zhu C, Nakamura H, Re N and Aquilanti V 1992 J. Chem. Phys. 971892

[19] Voronin A I, Karkach S P, Osherov V I and Ushakov V G 1976 Sov. Phys.-JETP 44465 Karkach S P 1993 J. Phys. A: Math. Gen. 266337

[20] Köppel H, Domcke W and Cederbaum L S 1994 Adv. Chem. Phys. 5759

[21] Longuet-Higgins H C 1961 Adv. Spectrosc. 2429

[22] Berry M V and Mount K E 1972 Semiclassical approximations in wave mechanics Rep. Prog. Phys. 35315

[23] Balian R and Bloch C 1974 Ann. Phys., NY 85514

[24] Fedoriuk M V 1993 Asymptotic Analysis (Berlin: Springer) ch 5

[25] Olver F W J 1974 Asymptotics and Special Functions (New York: Academic) ch 7

[26] Landau L and Lifshitz E 1967 Mécanique Quantique (Moscow: Mir) paragraph 25

[27] Baird L C 1970 J. Math. Phys. 112235 